\title{
Validación del segundo paso de la escalera analgésica de la OMS: ¿es necesario el uso de opioides débiles?
}

\author{
R.F. Rodríguez ${ }^{1}$ y M.L. Caicedo ${ }^{2}$ \\ ${ }^{1}$ Unidad de Especialistas en Dolor del Valle - UDOLOR. Universidad Libre Seccional Cali. Escuela de \\ Medicina. Corporación Comfenalco Valle Universidad Libre. Colombia. ${ }^{2}$ Universidad Libre Seccional Cali. \\ Colombia
}

Rodríguez RF, Caicedo ML. Validación del segundo paso de la escalera analgésica de la OMS: ¿es necesario el uso de opioides débiles? Rev Soc Esp Dolor 2013; 20(6): 285290.

\begin{abstract}
Objective: Compare the analgesic efficacy and tolerability of acetaminophen versus acetaminophen plus codeine in the relief of cancer-related pain

Methods: This 7-day, prospective, double-blind, randomized, parallel-group study was conducted with 36 patients with cancer pain. Outpatients were eligible for the study if they were aged $\geq 18$ years and had moderate to severe cancer-related pain. Eligible patients were randomly assigned to receive acetaminophen or acetaminophen plus codeine. The primary end point was the proportion of patients who achieved pain relief; the second end point was time to achieve relief pain.

Results: Of the 36 patients who participated, 20 received acetaminophen and 16 acetaminophen plus codeine. At baseline there were not differences between the groups. None of the between-group differences in response rates were significant ( $p$ $>0,05)$. The most common adverse effect in codeine group was constipation $(p=0,001)$.

Conclusion: Efficacy was comparable between acetaminophen and acetaminophen plus codeine over 7 days of treatment in this patients with moderate or severe cancer-related pain. Codeine was associated with significantly greater constipation.
\end{abstract}

Key words: Codeine. Acetaminophen. Analgesic ladder. Pain. Cancer.

Fuentes de financiación: Universidad Libre Seccional Cali y COLCIENCIAS.

Recibido: 02-04-2012

Aceptado: 28-06-2013

\section{RESUMEN}

Objetivo: Comparar la eficacia, seguridad y tiempo hasta el alivio del dolor por cáncer con acetaminofén solo versus acetaminofén más codeína con el fin de analizar si es necesario el segundo paso de la escalera analgésica de la OMS.

Métodos: Se realizó un ensayo clínico controlado, aleatorizado, doble ciego en mayores de 18 años, con dolor por cáncer de intensidad moderada o severa y Karnofsky $\geq 50 / 100$. Se excluyeron pacientes con antecedentes de drogadicción, insuficiencia renal o hepática y embarazo. Se dividieron en dos grupos, uno tratado con acetaminofén y otro con acetaminofén más codeína. Se realizó una evaluación inicial y controles diarios durante 7 días, evaluando analgesia, tiempo hasta el alivio del dolor y efectos colaterales. Se utilizaron las pruebas Chi cuadrado y Fisher para comparar proporciones, T y ANOVA para promedios. La comparación del tiempo en lograr alivio se realizó mediante una curva de Kaplan-Meier.

Resultados: Se asignaron veinte pacientes al grupo de acetaminofén y 16 al de acetaminofén más codeína. No se presentaron diferencias estadísticamente significativas en la eficacia de los dos tratamientos. El tiempo transcurrido entre la primera dosis y el alivio fue 41 minutos para acetaminofén y 34 minutos para codeína más acetaminofén $(p>0,05)$. El grupo de codeína presentó más estreñimiento $(p=0,001)$.

Conclusión: No se presentaron diferencias significativas en eficacia y tiempo hasta el alivio del dolor. El grupo de codeína presentó más estreñimiento que el de acetaminofén.

Palabras clave: Codeína. Acetaminofén. Escalera analgésica. Dolor. Cáncer.

\section{INTRODUCCIÓN}

El dolor es el síntoma principal en $70 \%(1,2)$ de los pacientes que padecen cáncer avanzado y en el $80 \%$ de 
los que reciben un tratamiento inadecuado del dolor (3-5). La terapia farmacológica con analgésicos es un componente esencial del tratamiento en virtud de que si se utilizan en forma adecuada, controlan el dolor en $80-90 \%$ de los pacientes (1,5-7).

Como guía para el adecuado manejo farmacológico del dolor por cáncer, la OMS diseñó una escalera analgésica de tres pasos, que en el 2006 cumplió 20 años de existencia $(1,6,8,9)$. Durante este periodo la escalera ha sido sometida a debate, elogiada por su simpleza y claridad y criticada por sus omisiones $(10,11)$. Si bien la escalera ha demostrado gran importancia, también ha sido criticada por autores que ponen en duda la necesidad del paso dos y abogan por el uso temprano de opioides potentes.

En el primer paso de la escalera se recomienda el uso de analgésicos no opioides, en el segundo el uso de opioides débiles solos o acompañados de no opioides y en el tercer peldaño opioides potentes solos o acompañados de no opioides.

Si bien los resultados de diferentes estudios son contradictorios (12-14), vale la pena preguntarnos si en el manejo del dolor por cáncer es necesario el uso de opioides débiles.

Por tal motivo se diseñó un ensayo clínico controlado con el objetivo de comparar la eficacia analgésica y seguridad de acetaminofén solo versus acetaminofén más codeína en alivio del dolor por cáncer.

\section{MATERIALES Y MÉTODOS}

Se realizó un ensayo clínico controlado, aleatorizado, doble ciego, en la Unidad de Dolor de la Clínica Universitaria de la Corporación Comfenalco Valle Universidad Libre. Se incluyeron pacientes mayores de 18 años con dolor de origen canceroso de intensidad moderada y severa, $>4$ en la escala visual análoga (EVA) y con Karnofsky mayor o igual a 50/100. Se excluyeron los que habían recibido codeína o acetaminofén durante las últimas 48 horas, con antecedentes de drogadicción, insuficiencia renal (creatinina $\geq 2 \mathrm{mg} / \mathrm{dl}$ ), bilirrubina $\geq 2 \mathrm{mg}$, los que recibían o habían recibido quimioterapia o radioterapia durante los últimos 10 días y los que padecían dolor de etiología no neoplásica de mayor intensidad que el dolor neoplásico; se excluyeron también mujeres embarazadas.

Se realizó un muestreo aleatorio por bloques. Cada bloque se conformó con 8 pacientes. Cada paciente recibió en orden de ingreso una caja numerada que contenía en su interior la cantidad de comprimidos necesarios para realizar el tratamiento analgésico. El empaquetamiento y numeración de los pastilleros fue realizado por un laboratorio contratado para tal fin. Los diferentes medicamentos eran iguales en sus características externas como color, forma, tamaño y sabor. El laboratorio entregó en sobres cerrados la identificación de los medicamentos que contenía cada caja numerada. Los comprimidos contenían $500 \mathrm{mg}$ de acetaminofén o $30 \mathrm{mg}$ de codeína más $500 \mathrm{mg}$ de acetaminofén.

Los pacientes y sus familiares recibieron orientación detallada en relación a cómo debían tomar los medicamentos, sobre los posibles efectos colaterales y qué hacer en caso de que se presentasen. Se dio un número telefónico para contacto médico permanente en caso de necesidad.

Se ordenó suspender otros analgésicos y medicamentos coadyuvantes antes de ingresar al estudio y se solicitó no consumir analgésicos diferentes a los del estudio durante el periodo de observación.

Para iniciar el manejo se administró un comprimido de cada medicamento con intervalos de cuatro horas, hasta completar 5 dosis diarias. En caso de no presentar alivio del dolor (dolor persistente de intensidad moderada o severa), la dosis fue duplicada.

En la evaluación inicial se realizó una historia clínica completa y una medición de la creatinina. Se realizaron controles médicos telefónicos cada 24 horas durante siete días. En los controles se indagó sobre la intensidad del dolor, adherencia al tratamiento y presencia de efectos colaterales. La primera y la última evaluación fueron presenciales; en ellas se realizó una evaluación clínica completa y se revisaron los pastilleros suministrados para cerciorarse del número de comprimidos consumidos.

Se consideró el tratamiento como eficaz cuando se logró suspender o disminuir la intensidad del dolor a un nivel leve (EVA: 0-4). Se evaluó el tiempo en que cada analgésico produjo alivio del dolor.

Para evaluar la seguridad de los medicamentos se evaluó la presencia de efectos adversos y el tiempo en que se presentaron.

La información recolectada fue manejada en un sistema de base de datos relacional realizada en Access. El análisis se realizó en Stata versión 11.

Se utilizó la prueba de Chi cuadrado y la prueba exacta de Fisher para comparar proporciones y la prueba $\mathrm{T}$ y ANOVA para comparar promedios. Se realizó una estratificación por edad, etiología del dolor y género y los potenciales confusores residuales se ajustaron en un modelo de regresión logística multivariado. La evaluación del tiempo en lograr alivio del dolor y el tiempo en presentarse los efectos adversos se realizó mediante un análisis de KaplanMeier. La significancia de los contrastes encontrados se evaluó con la prueba de Wilcoxon. Para el análisis multivariado se utilizó una regresión de Cox.

La muestra fue calculada con un margen de seguridad o confianza del $95 \%$ y un poder del $80 \%$, catalogando diferencias importantes a partir del $20 \%(15,16)$.

El proyecto y el consentimiento informado fueron revisados y aprobados por el Comité de Evaluación Ética de la Facultad de Salud de la Universidad Libre Seccional Cali y se obtuvo permiso de las directivas de la Clínica Universitaria de la Corporación Comfenalco Valle Universidad Libre. 


\section{RESULTADOS}

Se incluyeron 36 pacientes que ingresaron en el periodo comprendido entre enero de 2009 a diciembre 2011. Veinte fueron tratados con acetaminofén y 16 recibieron acetaminofén más codeína para el manejo del dolor por cáncer.

El diagrama de flujo de los pacientes se presenta en la figura 1.

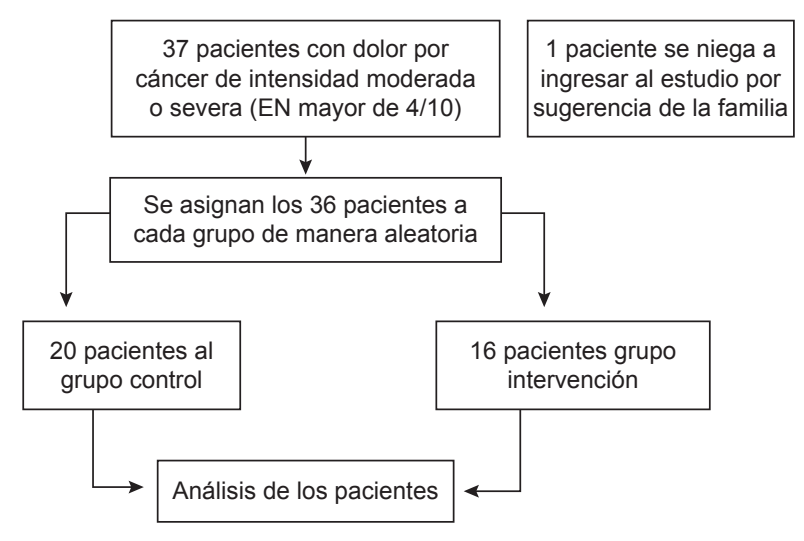

Fig. 1. Diagrama del flujo de pacientes a través de las diferentes fases del estudio.

Las variables demográficas y clínicas en condiciones basales se presentan en tabla I.

El tiempo trascurrido entre la administración de la primera dosis de medicamento y el momento en alcanzar alivio fue en promedio de 41 minutos para el acetaminofén y 34 minutos para la combinación de acetaminofén más codeína (Fig. 2).

En la tabla II se presentan los datos sobre tiempo a alivio y eficacia.

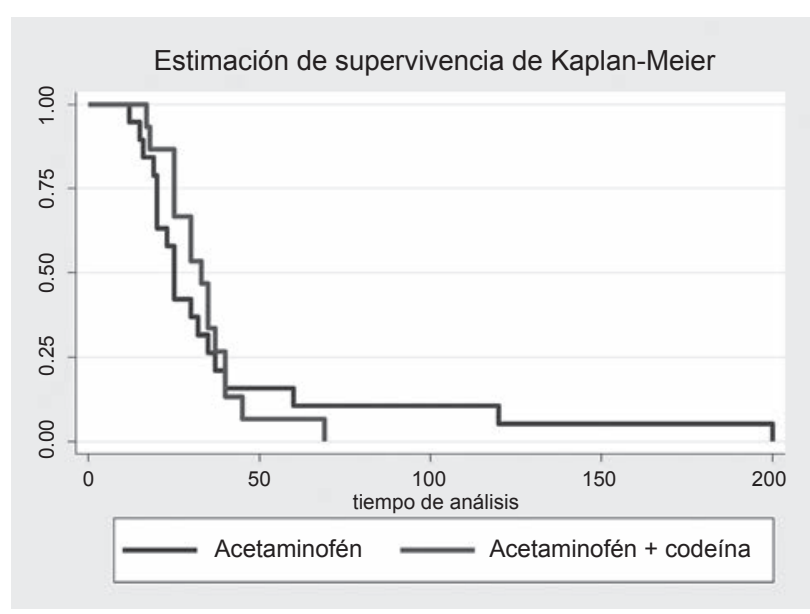

Fig. 2. Tiempo a alivio. Curvas de supervivencia de KaplanMeier según grupos de tratamiento.
TABLA I. CARACTERIZACIÓN DE VARIABLES DEMOGRÁFICAS Y CLÍNICAS EN CONDICIONES BASALES

\begin{tabular}{|c|c|c|}
\hline $\begin{array}{c}\text { Características } \\
\text { demográficas y clínicas }\end{array}$ & $\begin{array}{l}\text { Acetaminofén } \\
\quad(n=20)\end{array}$ & $\begin{array}{l}\text { Codeina } \\
(n=16)\end{array}$ \\
\hline Edad (años) $\chi \pm \mathrm{DE}$ & $58 \pm 14$ & $65 \pm 11$ \\
\hline Mujeres (\%) & 40 & 63 \\
\hline \multicolumn{3}{|l|}{ Diagnóstico (\%) } \\
\hline Mama & 15 & 20 \\
\hline Próstata & 10 & 13 \\
\hline Colon y recto & 10 & 20 \\
\hline Estómago & 15 & 20 \\
\hline \multicolumn{3}{|l|}{ Metástasis (\%) } \\
\hline Localizado & 5 & 7 \\
\hline Invasión regional & 21 & 13 \\
\hline Metástasis a distancia & 74 & 80 \\
\hline $\begin{array}{l}\text { Evolución dolor } \\
\text { (promedio días-rango) }\end{array}$ & $\begin{array}{c}163 \\
(10-1095)\end{array}$ & $\begin{array}{c}134 \\
(10-365)\end{array}$ \\
\hline $\begin{array}{l}\text { Intensidad del dolor } \\
\text { (promedio) }\end{array}$ & $7 / 10$ & $6 / 10$ \\
\hline \multicolumn{3}{|l|}{ Etiología del dolor (\%) } \\
\hline Somático & 35 & 53 \\
\hline Visceral & 60 & 40 \\
\hline Neuropático & 5 & 7 \\
\hline \multicolumn{3}{|l|}{ Síntomas asociados (\%) } \\
\hline Estreñimiento & 70 & 47 \\
\hline Debilidad & 80 & 87 \\
\hline Náuseas & 50 & 40 \\
\hline \multicolumn{3}{|l|}{ Karnofsky (\%) } \\
\hline Entre 50-70 & 60 & 67 \\
\hline$\geq 80$ & 40 & 33 \\
\hline
\end{tabular}

TABLA II. TIEMPO A ALIVIO Y EFICACIA

\begin{tabular}{lccc}
\hline $\begin{array}{c}\text { Tiempo alivio, } \\
\text { eficacia, éxito }\end{array}$ & $\begin{array}{c}\text { Acetaminofen } \\
(n=20)\end{array}$ & $\begin{array}{c}\text { Codeína } \\
(n=16)\end{array}$ & $p$ \\
\hline $\begin{array}{l}\text { Tiempo alivio } \\
\text { (minutos) } \pm \mathrm{DE}\end{array}$ & $\begin{array}{c}41 \pm 45 \\
(12-200)\end{array}$ & $\begin{array}{c}34 \pm 12 \\
(17-69)\end{array}$ & \\
\hline Eficacia - Alivio $(\%)$ & & & 0,082 \\
Todo el periodo & 40 & 69 & 0 \\
Parte del periodo & 55 & 19 & \\
Nunca & 5 & 12,5 & \\
\hline
\end{tabular}


Diferentes pacientes de ambos grupos presentaron una serie de efectos colaterales que se atribuyeron al consumo del analgésico administrado. La incidencia de efectos colaterales se presenta en la tabla III.

TABLA III. EFECTOS ADVERSOS ASOCIADOS A LA MEDICACIÓN

\begin{tabular}{lccc}
\hline $\begin{array}{c}\text { Efectos adversos } \\
\text { asociados a la } \\
\text { medicación }\end{array}$ & $\begin{array}{c}\text { Acetaminofén } \\
(n=20)\end{array}$ & $\begin{array}{c}\text { Codeína } \\
(n=16)\end{array}$ & $p$ \\
\hline Náuseas (\%) & 30 & 31 & 0,936 \\
Vómito (\%) & 20 & 19 & 0,925 \\
Estreñimiento (\%) & 0 & 44 & 0,001 \\
Diarrea (\%) & 20 & 0 & 0,058 \\
Disminución apetito (\%) & 10 & 12 & 0,813 \\
Disnea (\%) & 0 & 0 & \\
Malestar abdominal (\%) & 20 & 25 & 0,720 \\
Debilidad (\%) & 15 & 0 & 0,106 \\
Boca seca (\%) & 10 & 12,5 & 0,813 \\
Somnolencia (\%) & 30 & 44 & 0,393 \\
Mareo (\%) & 40 & 12,5 & 0,067 \\
Insomnio (\%) & 10 & 0 & 0,193 \\
Retención urinaria (\%) & 5 & 0 & 0,364 \\
Prurito (\%) & 0 & 25 & 0,018 \\
Sudoración (\%) & 10 & 19 & 0,451 \\
Alucinaciones (\%) & 0 & 0 & \\
\hline
\end{tabular}

TABLA IV. CAUSAS DE RETIRO DEL ESTUDIO

\begin{tabular}{lcc}
\hline \multicolumn{1}{c}{ Causas de retiro $(\%)$} & $\begin{array}{c}\text { Acetaminofén } \\
(n=20)\end{array}$ & $\begin{array}{c}\text { Codeína } \\
(n=16)\end{array}$ \\
\hline $\begin{array}{l}\text { Finaliza periodo } \\
\text { observación }\end{array}$ & 55 & 56 \\
$\begin{array}{l}\text { Efecto analgésico } \\
\text { inadecuado }\end{array}$ & 30 & 19 \\
$\begin{array}{l}\text { Efectos adversos } \\
\text { intolerables }\end{array}$ & 10 & 6 \\
\hline
\end{tabular}

De la tabla anterior se deduce que hubo diferencia estadísticamente significativa en relación al estreñimiento y prurito producido por la codeína, comparado con el acetaminofén.

Del estudio se retiraron 9 pacientes que recibieron acetaminofén y 7 tratados con codeína, la mayoría por no presentar control adecuado del dolor durante el periodo de observación; no hubo diferencias significativas al comparar las causas de retirada en los dos grupos (p: 0,4). Las causas de retirada se observan en la tabla IV.

Con los datos obtenidos se calculó la eficacia, la reducción absoluta del riesgo (RAR) y el número necesario a tratar (NNT) para aliviar un paciente, siendo los resultados presentados en la tabla $\mathrm{V}$.

\section{DISCUSIÓN}

No hubo diferencias estadísticamente significativas (DES) en cuanto a la eficacia en el efecto analgésico al comparar los dos grupos. Por medio de la curva de Kaplan Meier se comparó el tiempo en lograr alivio, sin encontrarse DES. El riesgo relativo (RR) compara el riesgo de padecer dolor en cada grupo. Un RR de 1,0 significa que el riesgo es igual en expuestos y no expuestos; en este caso el RR es 0,96 , muy próximo a 1,0 , lo que sugiere que no hay asociación entre la exposición a codeína y mayor alivio del dolor del dolor.

El NNT indica que se deberían tratar 40 pacientes con la combinación acetaminofén + codeína para obtener 1 caso de alivio adicional, al compararlo con el tratamiento realizado exclusivamente con acetaminofén.

A pesar del amplio uso de la escalera analgésica de la OMS durante más de veinte años, empiezan a aparecer resultados controvertidos en investigaciones que ponen en duda la necesidad de utilizar opioides débiles.

La mayoría de estudios que no encuentran DES se realizaron en modelos de dolor agudo postoperatorio. En una revisión sistemática de la literatura y el metaanálisis realizado por De Craen y cols. (17) utilizando 29 publicaciones, encontraron 19 estudios con administración de dosis única que demostraron que al administrar acetaminofén más codeína se obtenía un incremento del efecto analgésico de solo el $5 \%$, presentándose igual incidencia de efectos colaterales, mientras que los estudios con dosis múltiples mostraron una mayor incidencia de efectos colaterales en los

TABLA V. CÁLCULO DE LA EFICACIA, REDUCCIÓN ABSOLUTA DEL RIESGO Y NÚMERO NECESARIO TRATAR

\begin{tabular}{|c|c|c|c|c|c|}
\hline $\begin{array}{l}\text { Dolor con } \\
\text { acetaminofén } \\
\text { (Io) }\end{array}$ & $\begin{array}{c}\text { Dolor con } \\
\text { codeina } \\
\text { (Ie) }\end{array}$ & $\begin{array}{l}\text { Reducción } \\
\text { absoluta del } \\
\text { riesgo RAR } \\
(I o-I e) \\
\end{array}$ & $\begin{array}{c}\text { Riesgo relativo } \\
R R \\
(I e / I o)\end{array}$ & $\begin{array}{c}\text { Eficacia } \\
\text { RRR } \\
(1-R R) * 100 \% \\
(I o-I e / I o) * 100 \%\end{array}$ & $\begin{array}{c}\text { Número necesario } \\
\text { a tratar } \\
N N T \\
1 / R A R \\
\end{array}$ \\
\hline 40 & 37,5 & 2,5 & 0,96 & 4 & 40 \\
\hline
\end{tabular}


pacientes que recibieron la combinación de acetaminofén más codeína. Boureau y cols. (18) reportaron hallazgos similares en un estudio multicéntrico, aleatorizado doble ciego realizado en 198 pacientes con migraña.

Skjelbred y Lokken (19) realizaron un estudio doble ciego en 24 pacientes sometidos a amigdalectomía, comparando $400 \mathrm{mg}$ de acetaminofén frente a la misma dosis de acetaminofén mas $30 \mathrm{mg}$ de codeína, sin encontrar DES en el efecto analgésico, mientras que la incidencia de efectos colaterales fue significativamente mayor en el grupo que utilizó codeína. Arendt-Nielsen y cols. (20) realizaron un estudio controlado y aleatorizado comparando $1.000 \mathrm{mg}$ de paracetamol solo versus $1.000 \mathrm{mg}$ de paracetamol más $60 \mathrm{mg}$ de codeína utilizando un modelo de dolor inducido por láser, encontrando que la eficacia de la combinación era superior que el paracetamol solo, sin embargo la diferencia no fue estadísticamente significativa.

En el lado opuesto se encuentran los resultados que demuestran las ventajas de usar simultáneamente no opioides con opioides débiles. En una revisión sistemática realizada por Moore y cols. (21) para evaluar la analgesia de dosis orales únicas de acetaminofén como agente solo y en combinación con codeína para el dolor postoperatorio, encontraron 31 ensayos de paracetamol frente a placebo en 2.515 pacientes, 19 ensayos de paracetamol con codeína frente a placebo en 1.204 pacientes y 13 ensayos de paracetamol con codeína frente a la misma dosis de paracetamol como agente único en 874 pacientes; la comparación de $1.000 \mathrm{mg}$ de acetaminofén frente a placebo reportó un NNT de 3,6 (3,0-4,4), mientras que para 600/650 mg de acetaminofén el NNT fue 5,0 (4,1-6,9); para 600-650 mg de paracetamol más $60 \mathrm{mg}$ de codeína frente a placebo el NNT fue 3,1 (2,6-3,8). En las comparaciones directas de paracetamol con codeína frente a paracetamol como agente único, se observó que $12 \%$ de pacientes obtuvieron alivio mayor del $50 \%$ del dolor al agregarse $60 \mathrm{mg}$ de codeína, confirmando que la codeína produce un alivio adicional estadísticamente significativo. Kjaersgaard-Andersen y cols. (22) realizaron un estudio aleatorizado doble ciego, multicéntrico para evaluar la eficacia y seguridad de $1.000 \mathrm{mg}$ acetaminofén tres veces al día y de $1.000 \mathrm{mg}$ de acetaminofén con $60 \mathrm{mg}$ de codeína tres veces al día en 158 personas con artrosis de cadera que tenían en promedio 66 años de edad, por un periodo de 4 semanas; en forma significativa, se presentó mayor alivio del dolor en el grupo que utilizó codeína más acetaminofén, pero en este grupo se presentó la mayor incidencia de efectos colaterales. Teniendo en cuenta la edad de los pacientes, es importante considerar que se empleó una dosis inicial de codeína muy alta.

Podemos concluir diciendo que los resultados de este ensayo clínico controlado revelan que no existen diferencias estadísticamente significativas en la eficacia entre los dos grupos estudiados; tampoco hay diferencias en el tiempo a alivio. Las únicas diferencias con significancia estadística radican en que la codeína produce más estreñimiento y prurito que el acetaminofén, lo que pone en duda la necesidad de la existencia del segundo paso de la escalera de OMS.

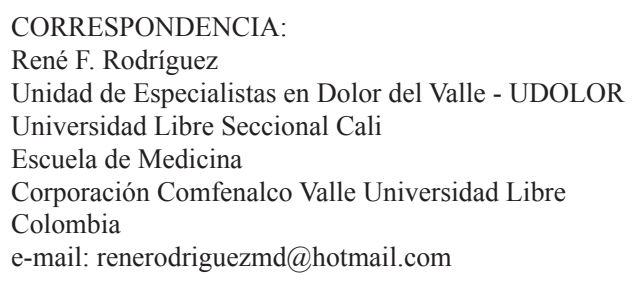

\section{BIBLIOGRAFÍA}

1. Cancer pain relief. Edit WHO. Genova; 1996. p. 3-37.

2. Rodríguez RF, Daza P, Rodríguez MF. Uso de buprenorfina transdérmica en el alivio del dolor por cáncer. Rev Col Anest 2006;34:253-7.

3. Twycross R, Bergh S, John S, Lewis K. Monitoring drug use in palliative care. Palliat Med 1994;8:137-43.

4. Payne R, Thomas J, Raj P. Dolor debido al cáncer. Epidemiología y enfoque terapéutico. En: Raj PP. Tratamiento práctico del dolor. Madrid: Mosby Year Book; 1994. p. 428-44.

5. Gómez SM, Ojeda MM. El dolor: analgésicos opiáceos: En: González MB. Tratado de medicina paliativa y tratamiento de soporte en el enfermo con cáncer. Madrid: Panamericana; 1996. p. 467-81.

6. Rodríguez RF. Manejo farmacológico del dolor en pacientes con cáncer. En: Rodríguez RF. Medicina del dolor y cuidados paliativos. Edit. Universidad Libre de Cali; 1998. p. 173-82.

7. OPS Cuidados Paliativos: Guías para el manejo clínico. Washington 1998. p. 39-46.

8. Rodríguez RF. Analgésicos opioides. En: Rodríguez RF. Medicina del dolor y cuidados paliativos. Edit. Universidad Libre de Cali; 1998. p. 199-204.

9. Fainsinger R, Schoeller T, Bruera E. Methadone in the management of cancer pain: A review. Pain 1993;52:137-47.

10. Brooks D, Gamble W, Ahmedzai S. A regional survey of opioid use by patients receiving specialist palliative care. Palliat Med 1995;3:229-38.

11. Nicholson B. Responsible prescribing of opioids for the management of chronic pain. Drugs 2003;63(1):17-32.

12. Przewlocki R, Przewlocka B. Opioids in chronic pain. Eur J Pharmacol 2001;429(I-3):79-91.

13. De Craen AJM, Di Glulio G, Lampe-Schoenmaeckers A, et al. Analgesic efficay and safety of paracetamol-codeine combinations versus paracetamol alone: A systematic review. BMJ 1996;313:321-5.

14. Boureau F, Joubert JM, Lassere V, et al. Double-blind comparison of an acetaminophen $400 \mathrm{mg}$-codeine $25 \mathrm{mg}$ combination versus aspirin $1000 \mathrm{mg}$ and placebo in acute migraine attack. Cephalagia 1994;14:156-61.

15. Miralles FP, González JM, Vaca JM. Investigación en el dolor experimental, medición y valoración del dolor. En: Torres LM. Medicina del Dolor. Barcelona: Editorial Masson S.A.; 1997. p. 19-33.

16. Dennis R. Como estimar el tamaño de la muestra en investigaciones con humanos. En: Llanos G. Epidemiología. Editorial Universidad Libre. Cali; 1998. p. 92-9. 
17. Rodríguez RF, Castillo JM, Castillo MP, et al. Codeine/Acetaminophen and Hydrocodone/Acetaminophen combination tablets for the management of chronic cancer pain in adults: a 23-day, prospective, double-blind, randomized, parallel-group study. Clinical Therapeutics 2007;29(4):581-7.

18. Rodríguez RF, Bravo LE, Castro F, et al. Incidence of weak opioids adverse events in the management of cancer pain: A double-blind comparative trial. Journal of Palliative Medicine. 2007;10(1):56-60.

19. Skjelbred P, Lokken P. Codeine added to paracetamol induced adverse effects but did not increase analgesia. Eur J Clin Pharmacol 1982;23(4):315-9.
20. Arendt-Nielsen L, Nielsen JC, Bjerring P. Double-blind, placebo controlled comparison of paracetamol and paracetamol plus codeine -a quantitative evaluation by laser induced pain. Eur J Clin Pharmacol 1991;40(3):241-7.

21. Moore A, Collins S, Carroll D, McQuay H. Paracetamol with and without codeine in acute pain: A quantitative systematic review. Rev Soc Esp Dolor 1998;5:104-11.

22. Kjaersgaard-Andersen P, Nafei A, Skov O, et al. Codeine plus paracetamol versus paracetamol in longer-term treatment of chronic pain due to osteoarthritis of the hip: A randomised, double-blind, multi-centre study. Pain 1990;43:309-18. 